\title{
A Suitable Polygonal Approximation for Laser Rangefinder Data
}

\author{
Laurent Charbonnier - Olivier Strauss \\ Laboratoire d'Informatique, de Robotique et de Micro-électronique de Montpellier \\ UMR 9928 - Université Montpellier II / CNRS \\ 161 Rue Ada, 34392 Montpellier cedex 5, France \\ Tel. (+33) 67-41-85-73, Fax (+33) 67-41-85-00, charbonn@lirmm.fr
}

\begin{abstract}
This paper deals with indoor mobile robot localization using 2D laser rangefinder data. An indoor environment is mainly made of connected or nonconnected planes (e.g., walls, doors, desks...). Therefore the laser rangefinder data are a set of points belonging to straight lines. The more suitable preprocessing of those data seems to be a polygonal approximation. However, classical approximation methods are not robust enough to provide a reliable local description.

We propose a new method to provide a robust polygonal approximation. It means accurate, reliable and repeatable detection of dominant points, such as angular points or break points. We thus obtain a set of angular points and break points (e.g., segments) whose detection depends neither on relative sensor location nor on measure noise. We present experimental results that demonstrate successful map building.
\end{abstract}

\section{Introduction}

The ability of a mobile robot to move in a partially known indoor environment depends fundamentally on the aptitude of its perception system to match sensor data to a database or to previous measurements. This aptitude depends on reliability, accuracy and repeatability of the pre-processing.

The exteroceptive data provided by vision sensors are often dense and accurate but not relevant enough nor structured to provide a reliable local map of the environment. Thus, a segmentation process must arrange in some coherent manner, information contained in several sensor measurements.

The exteroceptive sensor we use is a radial laser rangefinder. It provides a $2 \mathrm{D}$ description of the environment. In case of indoor environment, it is mainly made of connected or non-connected vertical planes such as walls, doors, desks... The laser rangefinder radially scans the environment in a plane parallel to the ground. Provided data are $3 \mathrm{D}$ points belonging to the intersection of the scanned plane and the set of environment vertical planes. Therefore, polygonal approximation is an appropriate model to give a compact and effective representation of such data.

Classical polygonal approximation methods are both based upon Cartesian properties of data and upon the hypothesis that measurement noise is uniform, homogeneous and identically distributed. Therefore, laser rangefinder data do not fit with this previous definition. So, the use of classical methods provides a polygonal approximation that is neither reliable nor accurate or repeatable.

In this paper, we propose a new polygonal approximation method that is suitable for laser rangefinder data. Section 2 presents the laser rangefinder we use and the data it provides. We focus on the measurement error. In Section 3, we review various segmentation methods of a set of coplanar points. We then propose a new approach. In Section 4 experimental results are presented which demonstrate the robustness of the proposed method.

\section{Laser rangefinder sensor}

The sensor we use is a radial laser rangefinder. The measurements are provided using time of flight method. The maximum range is 30 meters and the accuracy of the distance measurements $(10 \mathrm{~cm})$ is independent with regard to the distance between sensor and objects. The computation time is reduced compared to other $2 \mathrm{D} / 3 \mathrm{D}$ vision sensor. A system of increasing deflection made of a rotating mirror, provides a 270 degrees field of vision. The angular step up to 0.6 degrees.

This sensor radially scans a plane parallel to the ground and can provide eight $2 \mathrm{D}$ descriptions of the environment per second.

Sensor data consist in a sequence of coplanar points, provided into polar coordinates in the sensor frame. These data are not homogeneous in the Cartesian space. Due to electronic devices, the metric data (e.g., distance measures) are corrupted with a 
systematic sensor uncertainty (about $5 \mathrm{~cm}$ ). The reflection quality of the aimed surface generates a random noise which increases this imprecision. Therefore, any measurement below the range compulsory means the presence of an object.

The angular error is only due to the system and does not exceed 0.1 degrees. However, it can be noticed that nothing can be extrapolated between two angular sections. Thus, the angular zone separating two successive points remains an uncertainty zone.

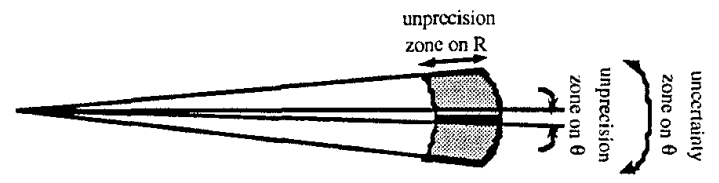

Figure 2-1: Sensor data

\section{Segmentation}

\subsection{Introduction}

An indoor environment is mainly made of connected or non-connected planes (e.g., walls, doors, desks...). The local map building is achieved with respect to the scanned points provided by the radial laser rangefinder. The whole map cannot be treated because of the volume and cost of storage data. The segmentation consists in obtaining a set of segments from a set of points. The more suitable pre-processing of those data seems to be a polygonal approximation.

A polygonal approximation of a set of coplanar points consists in finding a set of segments that fit at the best through the points. The main goal is then to locate the initial points and final points of each segment.

Classical methods are based upon Cartesian properties of data and upon the hypothesis that measurement noise is uniform and identically distributed. These methods can be classified in two kinds of approaches. The first one consists in searching characteristic points from the set of measurement points. The second one builds a piecewise linear approximation using a recursive method or an iterative method.

\subsection{Classical polygonal approximations}

A polygonal approximation of a digital curve made of $\mathrm{N}$ coplanar points $\mathrm{Pi}(\mathrm{i}=1$..N) belonging to straight lines, consists in finding the subset of those points located near the intersection of those lines. The first approach consists in detecting angular points directly through a measure of their singularity such as the curvature [TEH_89]. The algorithm must be able to exactly locate maxima of curvature on desired characteristic points. These methods extract several maxima and the treatment is more difficult. So it is necessary to adjust the horizon of the filter. Since the rangefinder data are corrupted with inhomogeneous noise, this kind of method cannot be applied. Moreover, in case of important noise, some characteristic points will not be detected. If a filter using good localization properties is chosen, this kind of method then gives accurate results but if the noise is not identically distributed or uniform, these methods are not reliable.

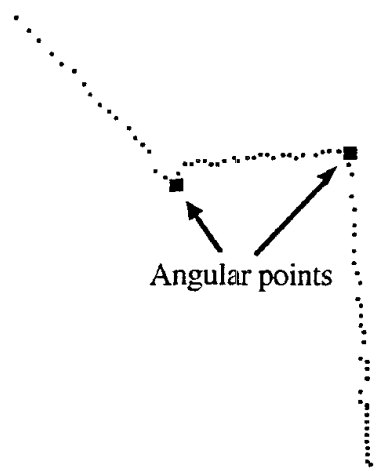

Figure 3-1: Angular points to be detect.

The second approach consists in obtaining a piecewise linear approximation of the digital curve subject to certain constraint of goodness. Two kinds of methods belong to the latter approach: iterative methods [WAL_84] and recursive methods [PAV_74].

Recursive methods are neither reliable nor accurate whatever input parameters are used. We shall restrict our discussion to iterative approach. These methods consist in scanning the sequence of data in the direction of increasing indices while a homogeneity criterion is verified. The initial point and final point of the current segment are the initial point and the latest point that satisfied the criterion (e.g., criterion<threshold). The detection qualities of these methods depend on the used criterion. It generally corresponds to the goodness fitting to the current linear approximation. Two kinds of problems can be encountered. The first one is the over-segmentation if the used threshold is too low. Due to this problem, the segmentation result is not accurate, there is a lot of segments which are non significant. In an other hand, if the chosen thresold is too high, segmentation is then biased (e.g., detection of angular points depends on the scanned direction). The segmentation result is then not accurate. Therefore, reliability of detection depends more on chosen criterion than on threshold. It means each angular point leads to the detection of characteristic point.

\subsection{Multi-criteria method}


The previous study shows that dominant points detection and iterative approximation method have complementary properties. The polygonal approximation we propose is much more suitable for laser rangefinder data. Classical methods repeatability depends on information about local noise, moreover these methods do not allow break points localization. In order to avoid problems encountered with classical methods (e.g., reliability and accuracy) the solution we propose consists in using the complementary properties of two classical methods to increase both reliability and accuracy. Problems involved in thresholding are solved in propagating a correct estimation of the local noise. The method we use is performed in four phases:

- measurement noise estimation. The first phase is dedicated to evaluate the a posteriori error variance on the set of data. This is performed using a Kalman filter controlled by a supervisor based upon qualitative calculus. This supervisor uses statistical tests to update the predicted error variance. This control measures the coherence between $a$ priori and $a$ posteriori variances [STR_92]. So, this phase evaluates the localization variance of each point.

- break points localization. A break point can be defined as a point before or after a gap in the sequence of data points. We define a gap as a distance between two succeeding points that is inconsistent with the uncertainty domain attached to them. Considering the study about the data error (phase 1), we assume that those domains are fuzzy patches [DUB_88]. The intersection of two fuzzy patches is used to define a measure of the possibility that can appear between two successive points.

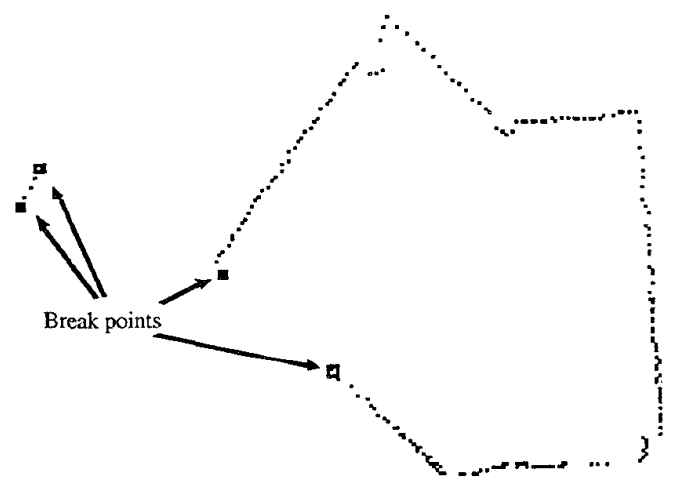

Figure 3-2: Crisp break points detection.

This measure is then used to compute the possibility of each point to be a break point. We then define a fuzzy subset of the break points. The membership value of each point in this subset is evaluated using this possibility measure. Hence, we apply the classical threshold $(0.5)$ to define the crisp break points as shown in Figure 3-2. However, the fuzzy value is used in the following phases.

- characteristic subset detection. Instead of finding directly the angular points, characteristics subsets are detected using an iterative method. The iterative method we use is based upon the Wall iterative method [WAL_84]. The efficiency of this algorithm is due to the use of a suitable criterion. This criterion is computed in summing up algebraic areas representing the segments fitting errors. The sum is then compared to a reference area linked to the distance between the current segment end points and to the local noise measure. This previous criterion is modified to take into account the possibility for a point to be to some extent a break point and so an angular point (Figure 3-3). Thus, we attach a fuzzy measure of goodness to the Wall criterion. The final decision is provided using the fuzzy disjunction of this fuzzy value and the membership value provided by the breakpoint detection (phase 2).

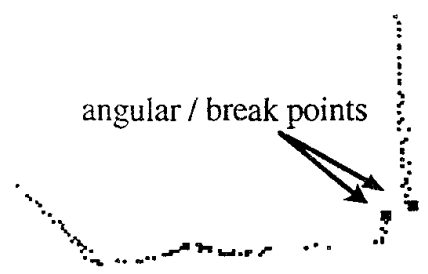

Figure 3-3: Angular points / break points.

The use of this method ensures that each angle point that induces detection of a characteristic point is a predecessor of this one in the sequence of data points $\mathrm{P}_{\mathrm{i}}$. This property leads us to consider as algorithm inputs first the sequence $P_{i}(i=1 . . N)$ and then, the reversed sequence $\mathrm{P}_{\mathrm{N}-\mathrm{i}+1}$. We thus obtain two sequence of characteristic points: the first one in scanning anti-clockwise the sequence $\mathrm{P}_{\mathrm{i}}$ (Figure 3-4), the second one in scanning clockwise (Figure 3-5). We then use an appropriate concatenation of those two sequences in order to define characteristic subsets as shown in Figure 3-6.

The repeatability of this method is ensured in using the error estimated by the variance error estimation process, previously computed.

Such characteristic subsets are characterized by the following property: each angle point to be detected belongs to at least one characteristic subset. On the opposite, there are characteristic subsets with no angular point. 


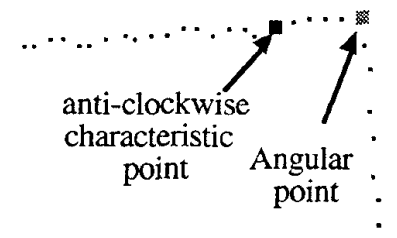

Figure 3-4: Anti-clockwise detection.

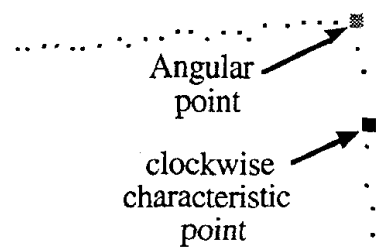

Figure 3-5: Clockwise detection.

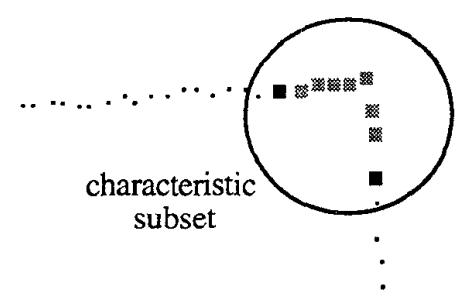

Figure 3-6: Characteristic subset extraction.

- angular point detection. We attempt to find all angular points in using a curvature estimate. This curvature is computed through an exponential filter designed to reduce quantization noise [CAS_90]. This filter is suitable because of its relevant localization properties. Angular points are detected in maximizing a curvature estimate into each characteristic subset. To remove subsets that contain no angular point, a fuzzy measurement of confidence is associated to each maximum detection in propagating measurement error in computation.

To reduce the number of obtained segments, an $a$ posteriori fusion phase is performed using fuzzy rules applied on criteria (e.g., disparate, heterogeneous, complementary). This phase is performed because the result of segmentation is not always optimal. It can include some mistakes because of aberrant points provided by the sensor. All these points are strored without any elimination. This phase just increase the robustness of the segmentation result.

We thus obtain a set of angular points and break points (e.g., segments) whose detection depends neither on relative sensor location nor on measure noise. With this method, we do assume the segmentation to be robust.

\section{Experimental results}

We have implemented and tested the multi-criteria segmentation method on our mobile robot VEGA, designed for moving in structured office environments. Robot control and data processing are performed in real time on transputer boards. We use laser rangefinder data as described in Section 2. We show in Figure 4-1 the set of data scanned by the sensor while the robot is exploring a room. The local map we build represents a $2 \mathrm{D}$ description of the environment. The proposed method first computes the measure noise in order to detect break points (Figure 4-2). Then, the procedure dedicated to provide the angular points scans the sequence of points using both directions: the first one is performed anticlockwise (Figure 4-3), the other one clockwise (Figure 4-4). All the characteristics detected points (e.g., break points and angular points) are shown in Figure 4-5. We can notice that each angle point of the room is included in one characteristic subset. Figure 4-6 shows the built local map after the multi-criteria method. The experimental results obtained with this system demonstrate successful map building.

\section{Conclusion}

2D laser rangefinder data are made of a sequence of coplanar points. The use of such a sensor in indoor mobile robotics requires compact and effective representation of those data. Polygonal approximation is a suitable model for indoor environments. However, most classical methods are based upon data Cartesian properties. Moreover, data noise is supposed to be a known uniform distribution. Laser rangefinders provide polar data whose error is partially known. Thus, no repeatability is ensured.

In this paper, a suitable polygonal approximation for laser rangefinder data has been presented. This method uses both qualities and drawbacks of two classical methods to ensure accuracy and reliability of the segmentation. Problems linked to break points are removed using a fuzzy uncertainty measure. A suitable analysis of the measurement error has been performed. An estimate of this error is propagated during all the process. This ensures the global robustness of the obtained segmentation. 


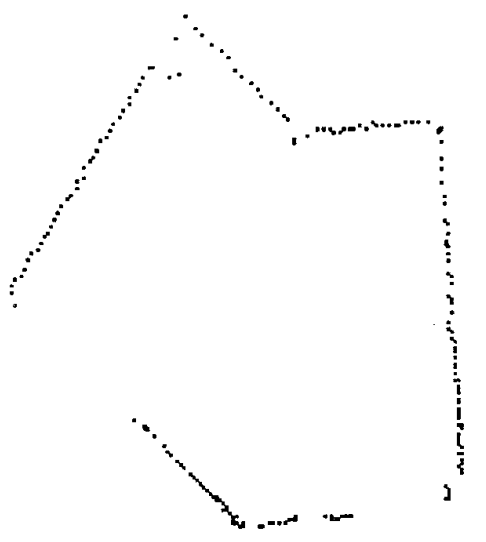

Figure 4-1: Laser rangefinder data example.

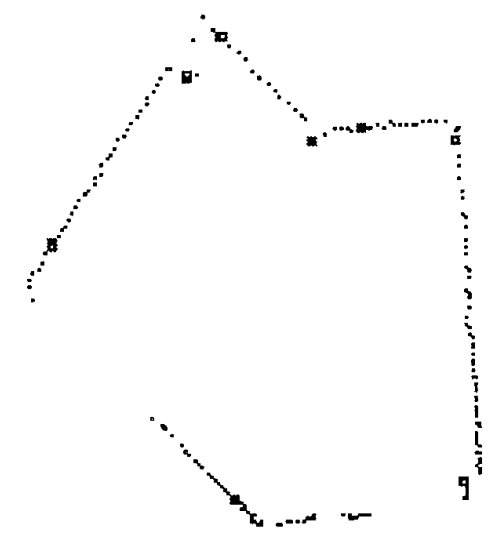

Figure 4-3: Anti-clockwise angular points detection

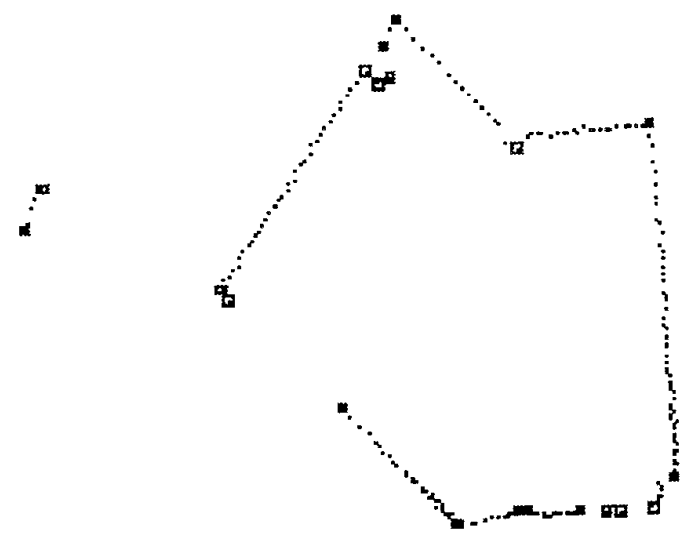

Figure 4-5: Characteristic points extracted.

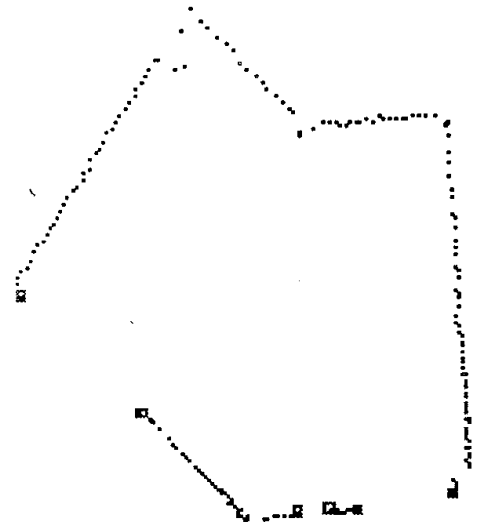

Figure 4-2: Break points detection.

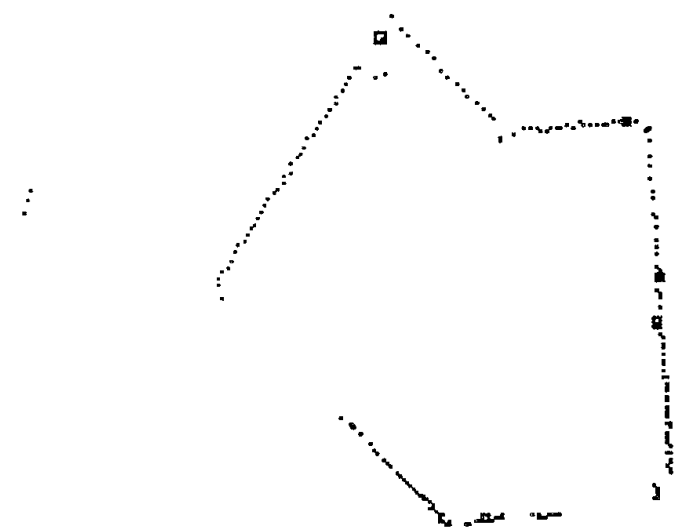

Figure 4-4: Clockwise angular points detection.

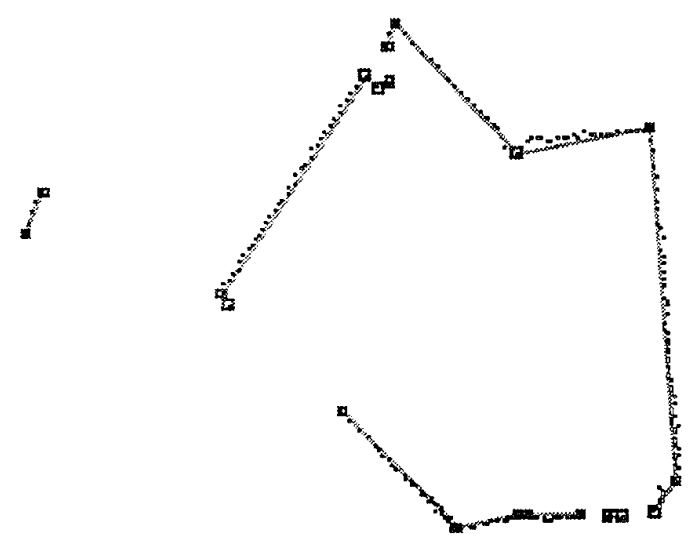

Figure 4-6: Segmentation result. 


\section{References}

[CAS_90] S. CASTAN, J. SHEN, J. ZHAO "Optimal filter for edge detection - methods and results" - pages 13-17 ECCV - April, 1990 - Antibes, France.

[DUB_88] D. DUBOIS, H. PRADE "Possibility theory - An approach to computerized processing of uncertainty" - Plenum Press - 1988 New York, USA.

[PAV_74] T. PAVLIDIS, S.L. HOROWITZ "Segmentation of plane curves" - IEEE Transaction on computer, vol 23, no 8, pages 860-870, 1974.

[STR_92] O. STRAUSS - "Perception de l'environnement par vision en lumière structurée: segmentation des images par poursuite d'indices" Thesis, UM2, January, 1992 - Montpellier, France.

[TEH_89] C.H. TEH, R.T. CHIN - "On the detection of dominant points on digitized curves" IEEE Transaction on pattern analysis and machine intelligence, vol 11, no 8, pages 859-872, August 1989.

[WAL_84] K. WALL, P.E. DANIELSSON "A fast sequential method for polygonal approximation of digitized curves" - Computer Vision, Graphics, Image Processing, vol 28, pages 220-227 - 1984. 